\title{
Design and Testing of a Fuel Consumption Eco-Driving Coach System for Truck Drivers based on Geolocation and BI Technologies
}

Stratakos loannis ${ }^{1}$, Sotiriou Panagiotis ${ }^{1}$, Perakis Harris ${ }^{1}$, Gikas Vassilis ${ }^{1}$, Spiliotakopoulos Konstantinos ${ }^{2}$, Pelekoudas Dimitris ${ }^{3}$

${ }^{1}$ School of Rural and Surveying Engineering, National Technical University of Athens, Athens, 15780, Greece

${ }^{2}$ Fortion Transport S.A., Patras, 26332, Greece

${ }^{3}$ Patras Informatics G.P., Patras, 26332, Greece

Correspondence to: Perakis Harris ${ }^{1}$ (hperakis@central.ntua.gr)

https://doi.org/10.31490/9788024845050-11

\begin{abstract}
Minimizing fuel consumption while maximizing driving performance (i.e., less travel time, increased safety and less gas emissions) is a key factor for the successful implementation of any transport system. In this regard, monitoring driving behaviour and converting this information into real-time advice to drivers can benefit seriously the performance of transport and mobility services.

This paper presents the design and implementation of a prototype system and a $\mathrm{BI}$ (Business Intelligence) model for fuel consumption optimization for heavy-duty vehicles. The system consists of an OBDH prototype, various external sensors (including GNSS/INS and environmental ones), and an on-purpose built FMS (Fleet Management System) bus logger. The paper revisits the concept of $\mathrm{Bl}$ and attempts knowledge transfer from the field of data analytics for business information to a pure engineering problem. Preliminary analyses using real truck trajectory data reveals and quantifies the effect of driving behaviour on fuel consumption while it indicates the potential of the system to transform into an integrated, self-trained driver coaching system.
\end{abstract}

Keywords: fuel consumption optimization, driver coaching GNSS/INS, Business Intelligence, FMS bus, automotive sensor technologies

\section{INTRODUCTION}

Fuel consumption is a critical parameter influencing transport efficiency and competiveness of the logistics sector, while contributes to global warming pollution. In recent years, advances in the automotive industry and in fuel processing technology have increased considerably fuel economy while decreasing pollutant emissions (Lipman, 2017). However, professional drivers' skills, habits and applied technique still have a great impact on fuel consumption. In the past, fuel-economy measures in relation to driving behaviour have been studied (Wang \& Boggio-Marzet, 2018; Keyvanfar et al., 2018) and various fuel consumption monitoring tools have been proposed (Perrotta et al., 2017; Schoen et al. 2019). Nevertheless, a fully automated truck driver coaching system to enable fuel consumption optimization is still missing. 
This study forms part of PEGASUS research project, the aim of which is to design, build and evaluate a novel professional truck driver coaching prototype (Gikas et al., 2021). The setup of the system relies on low-cost geolocation technologies (GNSS/INS), aiding sensors and parameters (e.g., vehicle speed, fuel use, accelerator pedal position) derived from the FMS bus (Fleet Management System). Once completed, the system will feature advanced parameterized $\mathrm{BI}$ analysis tools producing KPIs that would convert in the form of real-time advice to truck drivers to achieve optimal driving performance.

\section{PROTOTYPE DESIGN}

\section{a. Apparatus description}

Preliminary design investigations and testing have led to the development of the unified data acquisition and data transmission system that comprises three major components: (i) External sensors, (ii) FMS bus interface, and (iii) On-Board Data Hub (OBDH):

i. The external sensors of the system aim at providing critical information of the running state of the vehicle (position, velocity and heading), the environmental conditions (rain, ambient temperature / humidity), as well as measures that contribute to surroundings awareness capability (e.g., distance to heading vehicle) through using a camera / lidar sensor.

ii. An on purpose built measuring unit was designed and implemented to retrieve vehicle data from the FMS bus interface (i.e., subset of the CAN bus) (J1939-71, 2001; FMS Standard, 2002). As opposed to commercial of-the-shelf FMS bus loggers this approach enables better access and control in the low-level FMS bus subsystems.

iii. At the core of OBDH unit lies an Esp32 variant microcontroller. It acts as a high-rate datalogging system as well as data broadcasting unit through a WLAN connection to an onboard tablet.

Figure 1 illustrates the assembled $\mathrm{OBDH}$ prototype including the main subsystems and modules.

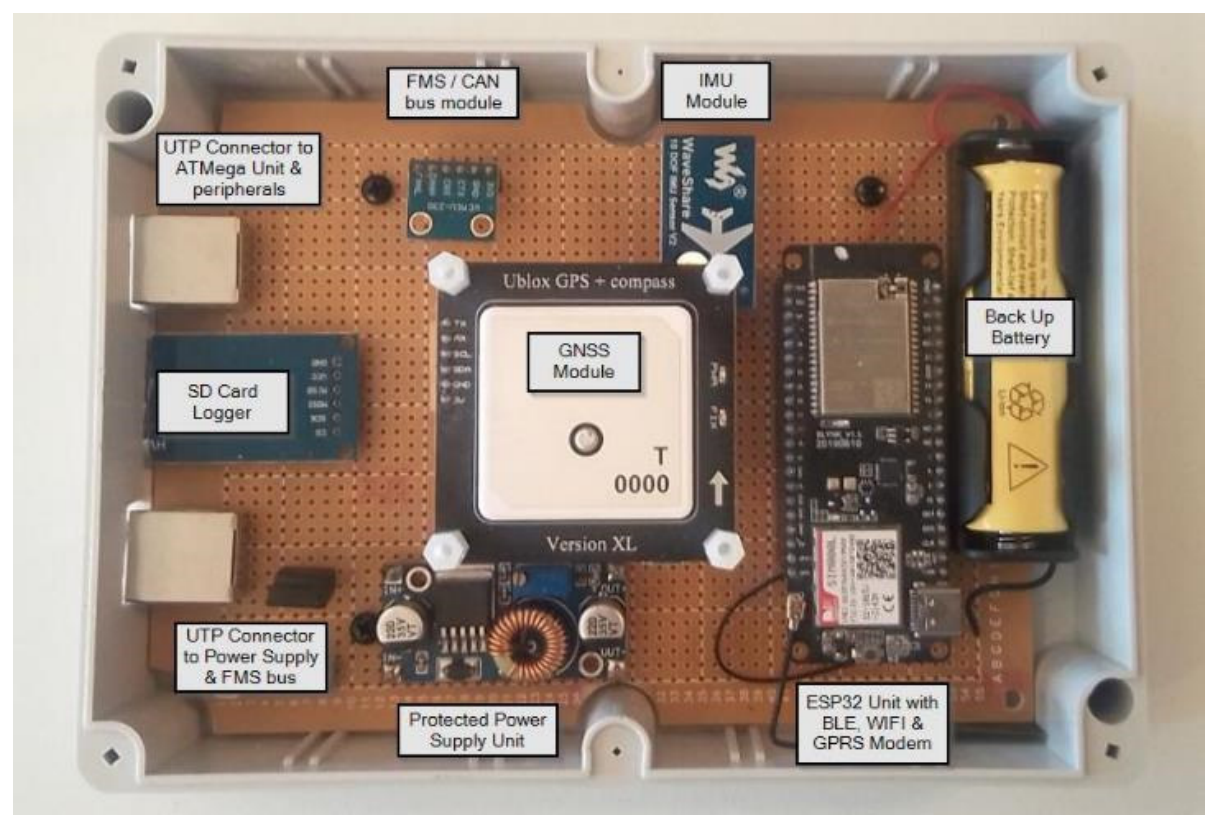

Fig. 1. Assembled OBDH prototype 


\section{b. BI modelling approach}

The proposed system fully exploits the benefits of $\mathrm{BI}$ technology. Contrary to the standard use of $\mathrm{Bl}$ in data analysis for business information (Inmon, 2005; Almeida, 2017; Liao, 2019), in this research the "model dimensions" represent the vehicle and operating condition parameters (i.e., time, trip chainage, discretized sets of engine speed, percentage of actual and requested engine torque values, horizontal curvature values, etc.), while the key "measurable" is the instantaneous and total fuel consumption. The $\mathrm{Bl}$ relational model consisted by appropriate dimension and transactional tables is illustrated in Figure 2. At an initial stage, analysis relies on a point-wise approach leading to a baseline KPI matrix which provides a map of the optimal driving conditions. Eventually, this approach enables expansion to a more detailed geometric feature-based analysis.

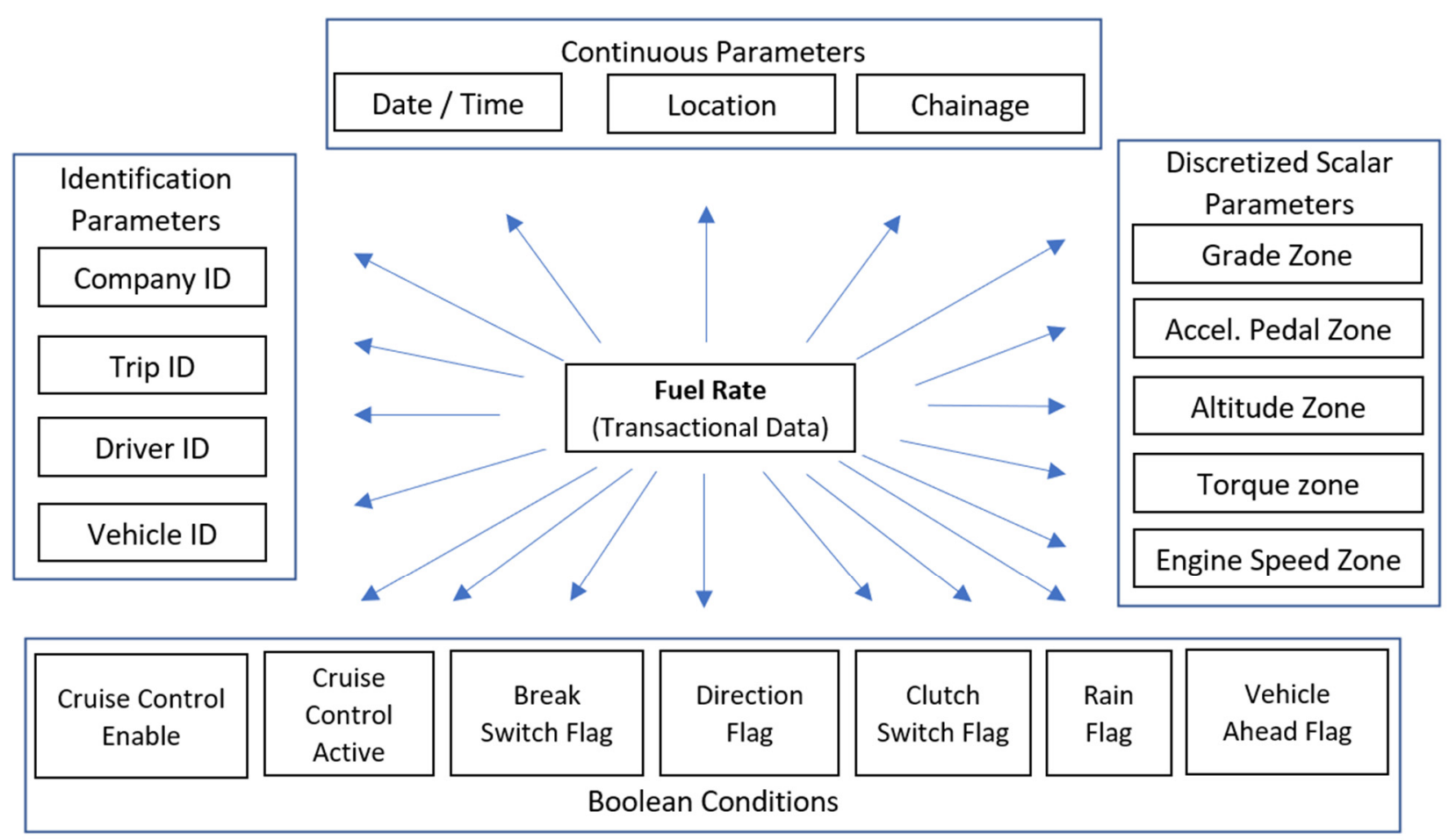

Fig. 2. BI Relational Model, consisting of a so-called "star schema" where the transactional data (fuel rate measurable) is joined with a primary key-foreign key relationship (arrows) to the dimension tables. In the discussed dataset, the Identification Parameters are excluded as they remain constant, while selected Boolean Conditions are used to filter out parts of the trip where the engine is strictly under load induced by the driver.

\section{PRELIMINARY DATA COLLECTION AND PROCESSING}

a. Engine Performance Evaluation

In order to demonstrate the role of the BI technology as part of a truck driver coaching system, this paper discusses the results from the analysis of a subset of a test trajectory using post processed data from a single trip. Figures 3 and 4 show the travel path and the elevation profile of the reference trip respectively. 


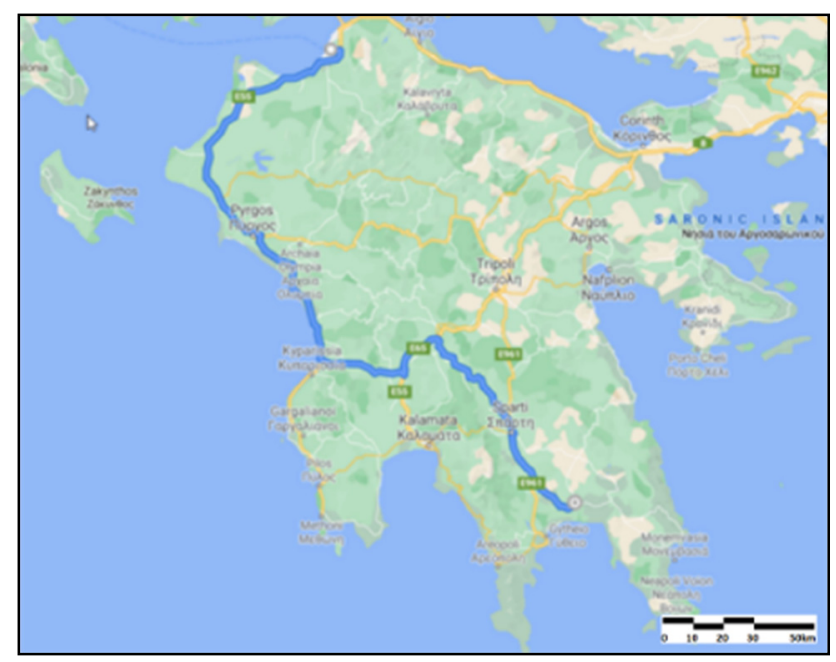

Fig. 3. Horizontal layout of the reference trip. Total distance travelled in both directions was $540 \mathrm{~km}$.

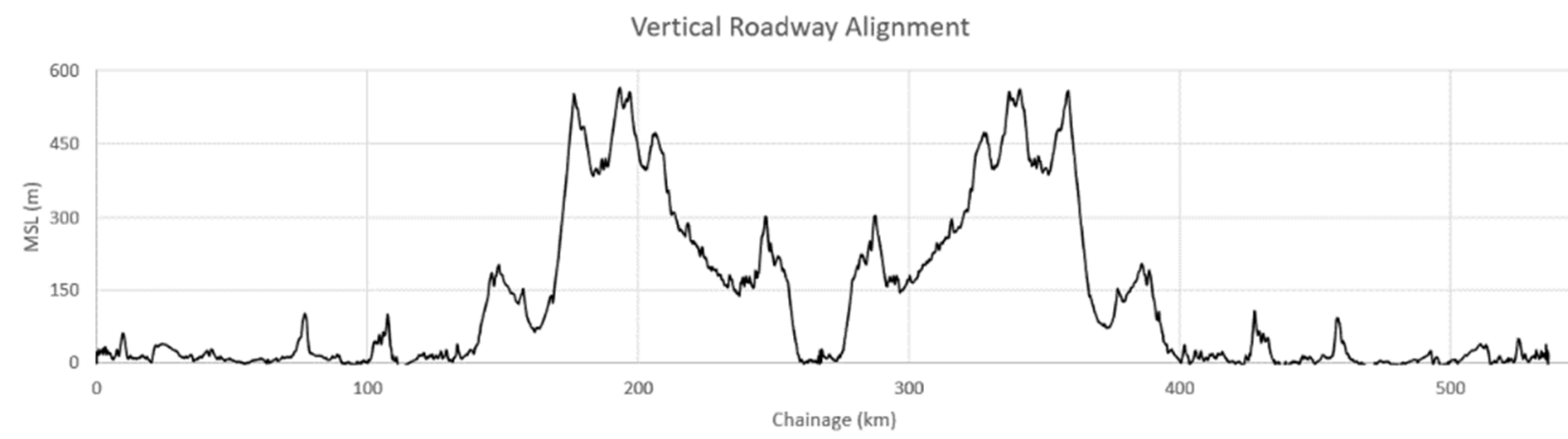

Fig. 4. Vertical profile of the travelled trip. The maximum elevation difference was $560 \mathrm{~m}$.

Utilizing the $\mathrm{BI}$ relational model presented in Figure 2, modelling fuel consumption optimization relies on GNSS, IMU and FMS bus data. Based on this information, the time history of fuel consumption against attained torque at specific engine speed ranges is reconstructed. In addition, geolocation and other information (i.e., Boolean conditions, grade and acceleration pedal position values of relational model) is used to filter out and exclude the vehicle data when travelling downhill, the segments while braking or the segments for which cruise control is activated. Therefore, the BI data model takes into account data samples only for those periods the engine is strictly under load induced by the driver. Consequently, the fuel rate is set to be a "measurable" in the BI system while the discretized set of engine speed and actual torque represent the "system dimensions". Thereby, a model of fuel consumption is obtained as a function of engine rpm (revolutions per minute) and actual torque (\%). Notably, the attained torque represents all the factors that translate into engine load, including cargo weight, vehicle weight, road grade, engine friction and varying driver's demand for engine power (e.g., due to just-in-time delivery requirements). Other parameters that may as well affect the overall trip consumption, such as gear selection preferences, tires condition, road condition or traffic, are also considered as they eventually yield specific combinations of torque, engine speed and consumption values. Finally, the BI system results in a pivot table, which represents the minimum fuel rate consumption for every combination pair of actual normalized torque and engine speed values (see Figure 3a). The resulting consumption map is validated against respective nominal parameters provided by the manufacturer while the envelope (red dashed line) is validated against 
independent CAN bus parameters obtained with a dedicated diagnostic device. Future evaluation against long-term fuel consumption will quantify system limitations enabling appropriate calibration.

In Figure 5a, a local minima valley at the centre of the graph can be observed. This indicates a typical situation of this type of graphs (Brahma et al., 2008; Vagg et al., 2012; Lang et al., 2013; Philips, 2015; Newman et al., 2015; Oglieve et al., 2017; Özener et al., 2018) that denotes the region of dimension values (i.e., torque and engine speed) that provide the most efficient fuel usage.

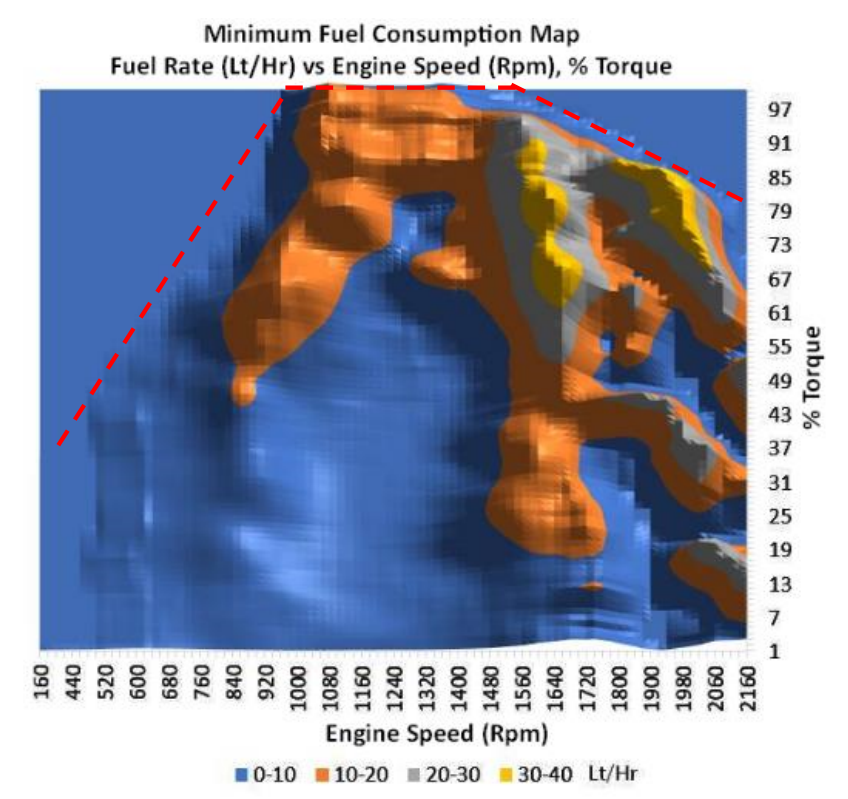

(a)

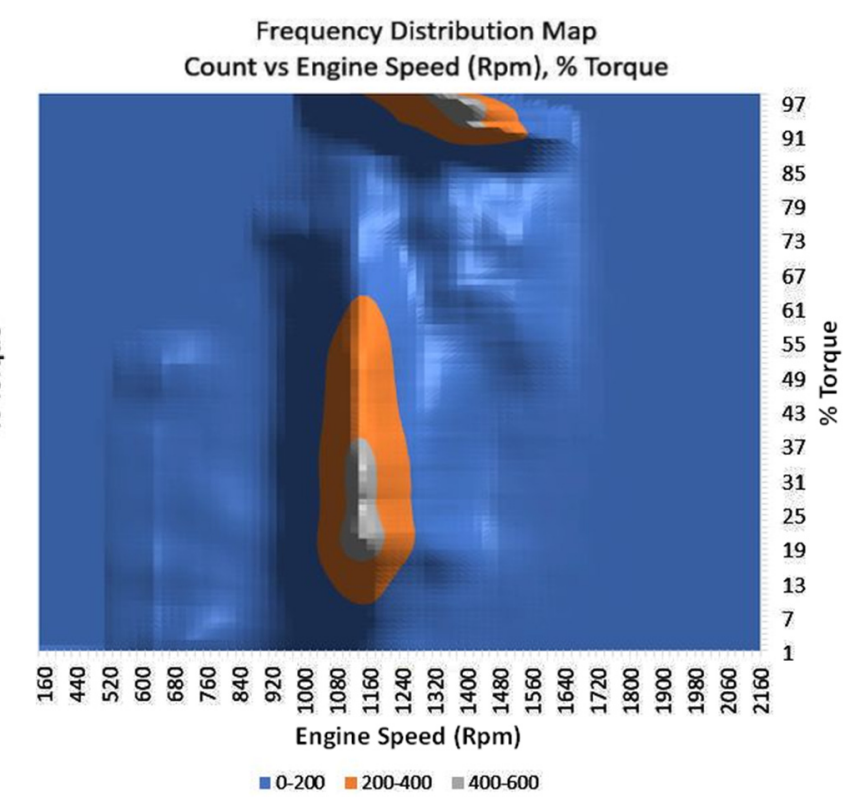

(b)

Fig. 5. Calculated fuel consumption map against engine speed and torque. The red dashed line denotes the envelope of maximum attainable torque by the engine (a). Frequency distribution map of fuel consumption against engine speed and torque indicating the driving behaviour (b).

\section{b. Driver's Performance Evaluation}

An alternate aggregation strategy of the $\mathrm{BI}$ pivoted report results in the count of fuel consumption values in each region. This representation provides a surface plot of the frequency distribution of fuel consumption concerning the engine operation on each of the areas shown in Figure 5(a). Clearly, Figure 5(b) reveals normal driver behaviour considering that the engine operation falls mainly within the optimum region of the fuel consumption map. The second region which corresponds to a high consumption area is located on the top zone of the frequency map at ca. $1400 \mathrm{rpm}$ and $97 \%$ of maximum torque, indicating a potential region of increased driving efficiency optimization margin.

\section{c. Fuel Economy Optimization}

Using the minimum (optimal) fuel consumption values shown in Figure 5(a) for the parts of the travelled route that correspond to the engine operation falling outside the high efficiency zone, a comparison of the actual fuel rate consumption against the potentially optimized one can be obtained. Figure 6 shows in red the actual fuel rate consumption for a part of the trip versus the travel path distance. In the same plot, the green line represents the optimized fuel rate consumption obtained for the same instantaneous values of torque and engine 
speed. The discrepancy between the two lines translates to an estimated $3 \%$ in fuel savings, suggesting the capabilities of the proposed methodology.

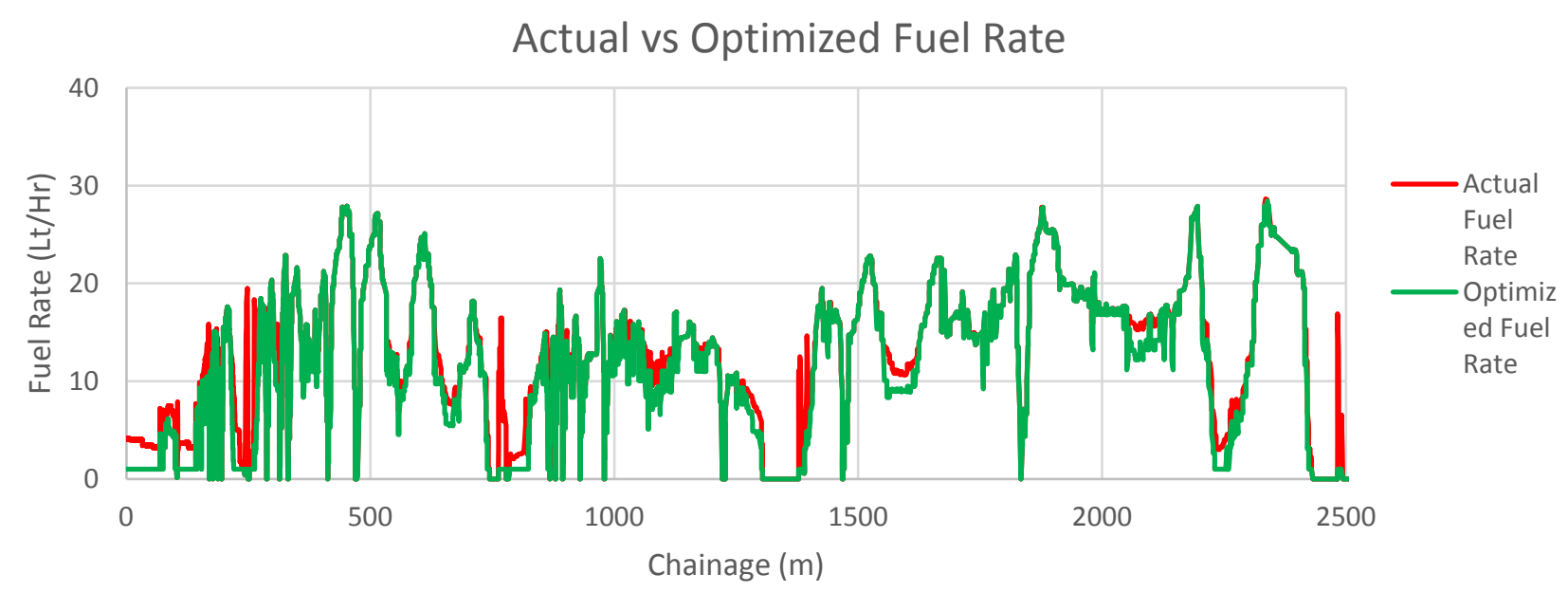

Fig. 6. Actual versus optimized fuel consumption rate.

\section{CONCLUSIONS AND FUTURE WORK}

The key idea of developing a novel approach for a truck driver coaching system is presented in this study. The system combines geolocation information derived from GNSS/IMU and vehicle FMS bus data fused using a BI system. More specifically, this study offers a BIbased technology model capable of producing optimal fuel consumption maps in regard to truck driver behaviour followed by the proof of concept of potential fuel consumption minimization margins. The significance of the proposed methodology lies in its ability to describe both the engine efficiency and the driver behavioural patterns, and their consequent correlations by implementing different aggregation strategies in a $\mathrm{BI}$ relational model. The proposed approach will be extended to compensate for the impact imposed by driving gear selection through real-time in-vehicle feedback to the driver in the form of vocal advices. Furthermore, the outcome of this work if combined with built in (computed within the system) information of road geometry (using the BI exports) can be used to provide fuel consumption information for the upcoming road segments in a timely manner. Such a capability would act proactively leading to additional fuel savings.

\section{FUNDING DECLARATION}

This research is co-funded by the European Regional Development Fund in the framework of the Operational Programme WESTERN GREECE 2014-2020.

\section{REFERENCES}

Almeida, F. (2017). Concepts and Fundaments of Data Warehousing and OLAP, ISSUU Publishing

Brahma, I., Sharp, M., and Frazier, T. (2008) Estimation of Engine Torque from a First Law Based Regression Model, SAE Technical Paper 2008-01-1014, doi:10.4271/2008-011014. 
Dekraker, P., Barba, D., Moskalik, A., and Butters, K. (2018) Constructing Engine Maps for Full Vehicle Simulation Modeling, SAE Technical Paper 2018-01-1412, 2018, doi:10.4271/2018-01-1412.

FMS-Standard (2002) Interface Description Version 01.00, FMS-Standard Working Group

Gikas, V., Spiliotakopoulos, K., Stratakos, I., Sotiriou, P., Perakis, H., Pelekoudas, D. (2021) Development of a prototype, self-trained truck driver coaching system based on geolocation, IoT and BI: Preliminary results and analyses, 7th International Conference on Models and Technologies for Intelligent Transportation Systems (MT-ITS), Heraklion, Greece (Accepted)

Inmon, W. H. Building the Data Warehouse, Fourth Edition. s.I. : Wiley Publishing Inc., 2005.

J1939-71 (2001) Vehicle Application Layer, SAE International

Keyvanfar A, Shafaghat A, Muhammad NZ, Ferwati MS. (2018) Driving Behaviour and Sustainable Mobility—Policies and Approaches Revisited. Sustainability, 10(4):1152. https://doi.org/10.3390/su10041152

Lang, D., Stanger, T., Re, L. (2013). Fuel efficient quasi optimal adaptive cruise control by control identification. Proceedings of the IEEE International Conference on Control Applications. 229-234. 10.1109/CCA.2013.6662763.

Liao, H. (2019). Intelligent Transportation Decision Analysis System Based on Big Data Mining. Journal of Physics: Conference Series. 1168. 032002. 10.1088/17426596/1168/3/032002.

Lipman, T. E. (2017). Emerging Technologies for Higher Fuel Economy Automobile Standards. Annual Review of Environment and Resources, 42(1), 267-288. https://doi.org/10.1146/annurev-environ-110615-085844

Newman, K., Kargul, J., and Barba, D., (2015) Development and Testing of an Automatic Transmission Shif Schedule Algorithm for Vehicle Simulation, SAE Int. J. Engines 8(3):1417-1427, 2015, doi:10.4271/2015-01-1142.

Oglieve, C., Mohammadpour, M., Rahnejat, H. (2017). Optimisation of the vehicle transmission and the gear-shifting strategy for the minimum fuel consumption and the minimum nitrogen oxide emissions. Proceedings of the Institution of Mechanical Engineers, Part D: Journal of Automobile Engineering. 231. 095440701770298. 10.1177/0954407017702985.

Özener, O., Özkan, M., Orak, E., Acarbulut, G. (2018) A fuel consumption model for public transportation with 3D road geometry approach. Thermal Science. 22. 158-158. 10.2298/TSCI1804211580.

Perrotta, F., Parry, T., and Neves, L. C., (2017) "Application of machine learning for fuel consumption modelling of trucks," 2017 IEEE International Conference on Big Data (Big Data), Boston, MA, 2017, pp. 3810-3815, doi: 10.1109/BigData.2017.8258382.

Phlips, P. (2015) Analytic Engine and Transmission Models for Vehicle Fuel Consumption 
Estimation, SAE Int. J. Fuels Lubr. 8(2):423-440, 2015, doi:10.4271/2015-01-0981.

Schoen, A., Byerly, A., Hendrix, B., Bagwe, R. M., Santos E. C. d., and Miled, Z. B., (2019) "A Machine Learning Model for Average Fuel Consumption in Heavy Vehicles," in IEEE Transactions on Vehicular Technology, vol. 68, no. 7, pp. 6343-6351, July 2019, doi: 10.1109/TVT.2019.2916299.

Vagg, C., Brace, C. J., Wijetunge, R., Akehurst, S. and Ash, L. (2012) Development of a new method to assess fuel saving using gear shift indicators. Proceedings of the Institution of Mechanical Engineers, Part D: Journal of Automobile Engineering, 226 (12). pp. 1630-1639. ISSN 0954-4070.

Wang Y, Boggio-Marzet A. (2018) Evaluation of Eco-Driving Training for Fuel Efficiency and Emissions Reduction According to Road Type. Sustainability, 10(11):3891. https://doi.org/10.3390/su10113891 